Pulsar Astronomy - 2000 and Beyond

ASP Conference Series, Vol. 202, 2000

M. Kramer, N. Wex, and R. Wielebinski, eds.

\title{
VLBI Neutron Star Astrometry: Techniques and Initial Results
}

\author{
Shami Chatterjee and James M. Cordes \\ Astronomy and Space Sciences, Cornell Universily, Ithaca, NY 14853
}

\begin{abstract}
We outline some new techniques being used and present initial results from a pulsar astrometry program at the NRAO Very Long Baseline Array (VLBA). We find a proper motion of $89.0 \pm 0.4 \mathrm{mas} / \mathrm{yr}$ and a preliminary parallax of $1.05 \pm 0.25$ mas for $\mathrm{B} 0919+06$.
\end{abstract}

Neutron stars are a population of galactic objects with surprisingly high measured velocities (up to $1500 \mathrm{~km} / \mathrm{s}$ ) and velocity dispersions. Very Long Baseline Interferometry (VLBI) offers a model-independent way to derive proper motions and parallaxes (out to $1-2 \mathrm{kpc}$ ) for radio pulsars: this complements timing and interstellar scintillation estimates of speeds and distances.

\section{VLBI Techniques}

Good VLBI imaging is possible in spite of very limited sampling of the Fourier plane due partly to the technique of self-calibration. However, self-calibration destroys absolute positional information, rendering it unusable for astrometry. As such, astrometric observations must be phase-referenced to a nearby ("nodding") calibrator with a known position using alternating scans. To allow phase connection at $1.4 \mathrm{GHz}$, we require scans no longer than 2 to 3 minutes and a nodding calibrator no more than $4-5^{\circ}$ away.

The VLBA correlator now supports pulsar gating, which blanks the offpulse signal and improves the signal to noise ratio by a factor of $\sim f^{-1 / 2}$, where $f$ is the gate duty cycle.

The major problem with phase referencing has been the differential ionosphere between the target and the nodding calibrator, which may cause scintillation and speckling due to multiple ionospheric paths, sidelobe amplitudes exceeding the real peak, and similar effects which prevent us from obtaining a reliable position. Several techniques are being explored to calibrate out this differential ionosphere: it is expected that some of these methods, possibly in combination, will allow accurate calibration.

\subsection{Using an in-beam calibrator}

A (possibly weak) extragalactic source located in the same primary beam as the target pulsar is a near-ideal situation, since one can self-calibrate on this weak source after standard phase referencing. The nodding distance is reduced from degrees to a few arcminutes, and the calibration is co-temporal instead of being interpolated across the 2-3 minute cycle time. In-beam calibration allows reliable astrometry to sub-milliarcsec precision: our results for $\mathrm{B} 0919+06$ use 
such an in-beam source (Fomalont et al. 1999). Unfortunately, finding sources which are both compact and in-beam is a challenging task.

\subsection{Using GPS data for ionospheric calibration}

The Global Positioning System (GPS) broadcasts signals at $1227.62 \mathrm{MHz}$ and $1575.42 \mathrm{MHz}$. The total electron content (TEC) along the line of sight to each GPS satellite can be inferred from the difference in time of arrival of time ticks at these two frequencies: this can be used map the ionosphere in near-real time.

We are currently using TEC maps provided at 11 minute intervals by SATLOC, a commercial firm, which allows calibration of the differential ionosphere between our target and nodding calibrator (Chatterjee 1999). The use of dual frequency GPS receivers at each VLBA antenna and ionospheric maps (at 2 hour intervals) archived at NASA's GSFC are also being investigated.

\subsection{Other methods}

The use of multiple nodding calibrators to allow interpolation of the calibration over the target area should work in principle, but preserving phase connection while cycling over multiple calibrators has proven difficult so far.

A modified self-calibration technique (Brisken et al. 1999) fits dispersive and non-dispersive components to the phases of multi-frequency data: this may be useful for strong pulsars, possibly in combination with GPS data.

\section{Results}

B0919+06: Combining two of our epochs with three epochs of earlier data, we get a proper motion $\mu_{\alpha}=18.1 \pm 0.2 \mathrm{mas} / \mathrm{yr}, \mu_{\delta}=87.1 \pm 0.3 \mathrm{mas} / \mathrm{yr}$, which is consistent with but much more precise than the catalog value of $\mu_{\alpha}=13 \pm 29$ mas $/ \mathrm{yr}, \mu_{\delta}=64 \pm 37 \mathrm{mas} / \mathrm{yr}$. We also obtain a preliminary parallax $\pi=$ $1.05 \pm 0.25$ mas, implying a distance of $1.0_{-0.2}^{+0.3} \mathrm{kpc}$ : this will be refined over further epochs.

Other pulsars: We find a two-epoch proper motion for B0823+26 of $\mu_{\alpha}=$ $77 \pm 5 \mathrm{mas} / \mathrm{yr}, \mu_{\delta}=-108 \pm 10 \mathrm{mas} / \mathrm{yr}$, consistent with catalog values. We also obtain two-point proper motions for $\mathrm{B} 1133+16$ and $\mathrm{B1642-03}$ : further epochs will improve these proper motions and possibly allow us to obtain parallaxes to these objects.

Acknowledgments. This research is supported in part by NSF grants AST 95-28391 and AST 99-19931. The National Radio Astronomy Observatory is a facility of the National Science Foundation operated under cooperative agreement by Associated Universities, Inc.

\section{References}

Fomalont, E. B., Goss, W. M., Beasley, A. J., Chatterjee, S. 1999, AJ, 117, 3025 Chatterjee, S. 1999, VLBA Scientific Memo Series (NRAO), No. 22

Brisken, W. F., Goss, W. M., Fomalont, E. B. 1999, AAS Meeting 194, 6210; and Brisken et al. in preparation. 